Association for Information Systems

AIS Electronic Library (AISeL)

Wirtschaftsinformatik Proceedings 2003

Wirtschaftsinformatik

September 2003

\title{
Automatisierte Koordinationsmechanismen zur Ausgestaltung komplexer Verträge im Rahmen mediatisierter Verhandlungsprozesse
}

Andreas Fink

Universität Hamburg, fink@econ.uni-hamburg.de

Follow this and additional works at: http://aisel.aisnet.org/wi2003

\section{Recommended Citation}

Fink, Andreas, "Automatisierte Koordinationsmechanismen zur Ausgestaltung komplexer Verträge im Rahmen mediatisierter Verhandlungsprozesse" (2003). Wirtschaftsinformatik Proceedings 2003. 16.

http://aisel.aisnet.org/wi2003/16

This material is brought to you by the Wirtschaftsinformatik at AIS Electronic Library (AISeL). It has been accepted for inclusion in Wirtschaftsinformatik Proceedings 2003 by an authorized administrator of AIS Electronic Library (AISeL). For more information, please contact elibrary@aisnet.org. 
In: Uhr, Wolfgang, Esswein, Werner \& Schoop, Eric (Hg.) 2003. Wirtschaftsinformatik 2003: Medien - Märkte - Mobilität, 2 Bde. Heidelberg: Physica-Verlag

ISBN: 3-7908-0111-9 (Band 1)

ISBN: 3-7908-0116-X (Band 2)

(C) Physica-Verlag Heidelberg 2003 


\title{
Automatisierte Koordinationsmechanismen zur Ausgestaltung komplexer Verträge im Rahmen mediatisierter Verhandlungsprozesse
}

\author{
Andreas Fink \\ Universität Hamburg
}

Zusammenfassung: In dem Beitrag werden Koordinationsmechanismen zur Ausgestaltung komplexer Verträge im Rahmen mediatisierter Verhandlungsprozesse beschrieben und experimentell untersucht. Unter der Annahme einer Formalisierbarkeit des Vertragsraums werden bilaterale Verhandlungsprozesse agentenbasiert vollautomatisiert durchgeführt. Hierbei entwickelt ein Mediator sukzessive veränderte Vertragsvorschläge, die von den Verhandlungspartnern gemäß robuster Akzeptanzkriterien beurteilt werden, ohne dass die eigentlichen Nutzeneinschätzungen der Verhandlungspartner offen gelegt werden. Trotz dieser Einschränkung resultieren die Verhandlungsprozesse in Verträgen hoher Güte. Eine hierbei auftretende Ausprägung des Gefangenendilemmas wird durch Vorgabe und Verifikation eines bestimmten Grades an kooperativem Verhalten gelöst.

Schlüsselworte: elektronische Verhandlungen, e-Negotiations, e-Contracting, mediatisierte Verhandlungsprozesse

\section{Einführung}

Im Sinne eines methodologischen Individualismus ist in ökonomisch geprägten Systemen von eigennützig handelnden Akteuren auszugehen, die jedoch über geeignete Formen der Kooperation gemeinsame Erfolge erzielen können. Eine entsprechende Koordination erfolgt insbesondere auf der Grundlage von Verträgen, womit die Kooperation zwischen autonomen Akteuren geregelt wird. Verträge können durch Verhandlungen ermittelt werden, wobei entsprechende Transaktionskosten anfallen. Das Zustandekommen von Verträgen im Rahmen interaktiver (personeller) Vertragsverhandlungen ist gegebenenfalls einem gewissen „Geschick“ der Verhandlungspartner unterworfen, weshalb Raiffa auch von „The Art and Science of Negotiation" spricht [Raif82]. Die formal-wissenschaftliche Betrachtung von Verhandlungen bezog sich in der Vergangenheit primär auf Verhandlungen zwischen zwei oder wenigen Akteuren (Negotiations). Bilaterale Verhandlungen hinsichtlich der Festlegung lediglich eines Attributes (Issues) - etwa des Preises für den Austausch eines Gutes - subsumiert man in der Regel unter 
dem Begriff Bargaining. Hierbei kommen zurückgehend auf grundlegende Modelle und Erkenntnisse insbesondere von Nash [Nash50; Nash53] sowie Rubinstein [Rubi82] hauptsächlich spieltheoretische Konzepte zum Einsatz [Muth99; Nape02; Roth85]. Ökonomische Transaktionen und entsprechende vertragliche Regelungen sind hingegen häufig durch eine Vielzahl interdependenter Attribute charakterisiert, weshalb man dann auch von komplexen Verträgen spricht. Beispiele sind Verträge in Liefernetzwerken (Supply Chains), die Festlegungen nicht nur zu Preisen sondern etwa auch zu Terminen, Mengen, Abläufen usw. enthalten [VoSc02]. Aufgrund von Interdependenzen - wie komplementären und substitutiven Beziehungen - sollten einzelne Vertragsattribute dann nicht isoliert betrachtet und verhandelt werden, sondern einer integrativen Behandlung unterliegen.

Im Kontext einer zunehmenden informationstechnischen Abwicklung der unternehmensinternen und unternehmensübergreifenden Koordination von Geschäftsprozessen (Electronic Business) ergeben sich grundsätzliche Potenziale sowie ein entsprechender Bedarf für eine möglichst weitreichende Automatisierung der Ausgestaltung komplexer Verträge mittels so genannter elektronischer (automatisierter) Verhandlungen. Dies gilt insbesondere im Zusammenhang mit mittel- bis längerfristigen Geschäftsbeziehungen zwischen eigenständigen Unternehmen, die von einer informationstechnisch unterstützten hybriden Koordinationsform - mit Märkten und Hierarchien als den Extrempunkten des entsprechenden Spektrums im Sinne der ,Move to the Middle“-Hypothese in besonderem Maße profitieren können [Clem+93; BaBr93]. Vor dem Hintergrund des von Mertens propagierten Langfristziels einer „sinnhaften Vollautomation“ [Mert95] stellen sich gerade für die Wirtschaftsinformatik zentrale Aufgaben hinsichtlich der Erklärung und Gestaltung entsprechender automatisierter Koordinationsmechanismen. Dabei bietet sich die Repräsentation der Verhandlungspartner als softwarebasierte Agenten an [Jenn+01; Krau01; RoZ194]. Eine wesentliche Voraussetzung für eine Vollautomation von Verhandlungen zwischen Agenten ist die Formalisierung von Verträgen. Das heißt, die Verhandlungspartner müssen eine formale Definition der Menge prinzipiell zulässiger Verträge angeben können. (In dieser Arbeit werden somit Verhandlungen mit ex-ante unstrukturierten Vertragsoptionen und nichtquantitativen Nutzenbewertungen nicht betrachtet.) Eine explizite Aufzählung möglicher Verträge sowie eine vollständige Angabe der Nutzenfunktion für alle Verträge sind nicht vorauszusetzen - die Agenten müssen lediglich in der Lage sein, auf Anfrage den Nutzen eines potenziellen Vertrags im Vergleich einzuschätzen. Die Nutzeneinschätzungen sind als private Informationen zu betrachten, die ein Verhandlungspartner in der Regel nicht offen legen möchte - lediglich ein Akzeptieren oder Nicht-Akzeptieren eines Vertrags wird geäußert. Dabei ist von einem strategischen Verhalten auszugehen, so dass Äußerungen nicht eo ipso als wahrhaftig angenommen werden können.

Ziel dieses Beitrags ist die Erklärung und Gestaltung automatisierter Koordinationsmechanismen zur Ausgestaltung komplexer Verträge (,e-Contracting “) unter den beschriebenen Randbedingungen. Die Untersuchung baut auf Ansätzen einer 
Forschungsgruppe am Massachusetts Institute of Technology / New England Complex Systems Institute [Klei+02a; Klei+02b] zur mediatisierten Abwicklung von Verhandlungsprozessen auf. Im folgenden Abschnitt wird das zugrunde gelegte Modell (Protokoll) des Verhandlungsprozesses in generischer Form mit verschiedenen Konkretisierungen - im Sinne alternativer Ausgestaltungen - dargestellt. Die Ergebnisse diesbezüglicher experimenteller Untersuchungen sind in Abschnitt 3 dokumentiert. Die sich hieraus ergebenden idealtypischen Konfigurationen des Verhandlungsprozesses werden in Abschnitt 4 aus einer spieltheoretischen Sichtweise näher betrachtet, bevor in Abschnitt 5 wesentliche Erkenntnisse zusammengefasst und offene Forschungsfragen diskutiert werden.

Elektronische Verhandlungen stellen ein aktuell in breiter Form behandeltes Forschungsgebiet dar [BeSe97; Bich+02; Jenn+01; Kers02; Kers03; Lomu+03; Rebs01]. Über die Definition elektronischer Verhandlungen sowie auch die Abgrenzung zwischen Auktionen und Verhandlungen als den zentralen marktlichen Koordinationsformen herrscht noch keine volle Übereinstimmung [Bich+02; Kers+00; Lomu+03]. Ohne im Rahmen dieser Arbeit näher auf die noch nicht abgeschlossene Erschließung des Gebiets einzugehen, kann dieser Beitrag hinsichtlich wesentlicher Aspekte folgendermaßen eingeordnet werden:

- Es werden potenziell wertschaffende (,integrative“ / „Win-Win“) Kooperationen betrachtet, die im Zusammenhang mit komplexen multiattributiven Verträgen stehen (im Gegensatz zu ,distributiven“ Verhandlungen zur Preisbildung im Rahmen von Nullsummenspielen).

- Die Verhandlungspartner nehmen symmetrische Rollen ein (im Unterschied zu einer expliziten Unterscheidung in Anbieter und Nachfrager).

- Im Mittelpunkt stehen bilaterale Verhandlungen (im Gegensatz zu Ansätzen zu multiattributiv ausgerichteten multilateralen Verhandlungs- bzw. Auktionsformen, die direkt auf eine Koordination zwischen vielen Akteuren - insbesondere hinsichtlich einer Abstimmung zwischen Angebot und Nachfrage - abzielen [Pete00; Strö02]). Der beschriebene Ansatz ist grundsätzlich auch auf multilaterale Verhandlungen erweiterbar.

- Im Verhandlungsprozess werden komplexe Verträge sowie simultane Vertragsvariationen in beliebiger Ausprägung betrachtet. Dies steht im Gegensatz zur sequenziellen Abhandlung einzelner Vertragsattribute gemäß einer festzulegenden Reihenfolge (Agenda) [Fati+03, Wede00].

- Ziel ist die agentenbasierte Vollautomatisierung von Verhandlungsprozessen (im Gegensatz zu Szenarien, bei denen menschliche Akteure hinsichtlich der Informationsbereitstellung und -verarbeitung im Ablauf von Verhandlungsprozessen unterstützt werden sollen (,Negotiation Support Tools“, „ENegotiation Media“), der eigentliche Verhandlungsablauf hingegen maßgeblich durch manuelle Entscheidungen beeinflusst wird). 


\section{Modell des Verhandlungsprozesses}

Der in diesem Beitrag betrachteten Problemstellung liegen die folgenden Annahmen zugrunde: Zwei Akteure $A_{1}$ und $A_{2}$ stehen vor der Aufgabe, mittels bilateraler Verhandlungen einen wertschaffenden Vertrag abzuschließen. Die Menge der prinzipiell zulässigen Verträge ist über einen formal definierten Vertragsraum $C=$ $\{c \mid c$ ist ein zulässiger Vertrag $\}$ gegeben, über den bereits ein beiderseitiges Einvernehmen erzielt wurde. Die Akteure $A_{1}$ und $A_{2}$ beurteilen Verträge gemäß ihrer jeweiligen Präferenzen anhand von Nutzenfunktionen $f_{1}: C \rightarrow \mathrm{R}$ bzw. $f_{2}: C \rightarrow \mathrm{R}$. (Für einen Akteur auf keinen Fall akzeptable Verträge führen zu einer negativen Nutzenbewertung; damit ergibt sich ein Reservationsniveau von Null.) Diese Nutzeneinschätzungen sind als private Informationen zu betrachten, die ein Verhandlungspartner nicht offen legen möchte. Ziel der Akteure ist jeweils die opportunistische Maximierung des Eigennutzens über einen individuell möglichst guten, beiderseitig akzeptierten Vertragsabschluss. Aufgrund der Komplexität des Vertragsraums ist eine vollautomatisierte Abwicklung des Verhandlungsprozesses angebracht. Zur Abwicklung des Verhandlungsprozesses kommt ein Mediator $M$ zum Einsatz, dessen Aktionen vollständig offen gelegt werden können. $M$ hat keinen Einblick in die Nutzenfunktionen oder in sonstige private Informationen der Akteure; besondere Vertrauensverhältnisse werden nicht vorausgesetzt. Der grundsätzliche Verhandlungsprozess wird ähnlich wie in herkömmlichen Verhandlungsabläufen in Anlehnung an [Klei+02a] durch eine wiederholte Ausführung der folgenden Abfolge im Sinne eines strukturierten Protokolls gestaltet:

1. Vorschlag eines potenziellen Vertrags durch $M$;

2. Vorlage des potenziellen Vertrags an $A_{1}$ und $A_{2}$ und jeweilige Akzeptanz- oder Ablehnungsentscheidungen;

3. (Vorläufige) Anerkennung des Vertrags im Falle einer beiderseitigen Akzeptanzentscheidung.

Dieser Ablauf ist in dem Sequenzdiagramm in Abbildung 1 veranschaulicht. Die wesentlichen Variationspunkte dieses generischen Verhandlungsprozesses sind der Vertragsraum $C$, der hiervon abhängige Mechanismus des Mediators $M$ zur Generierung von Vertragsvorschlägen sowie die Akzeptanzkriterien der Verhandlungspartner $A_{1}$ und $A_{2}$.

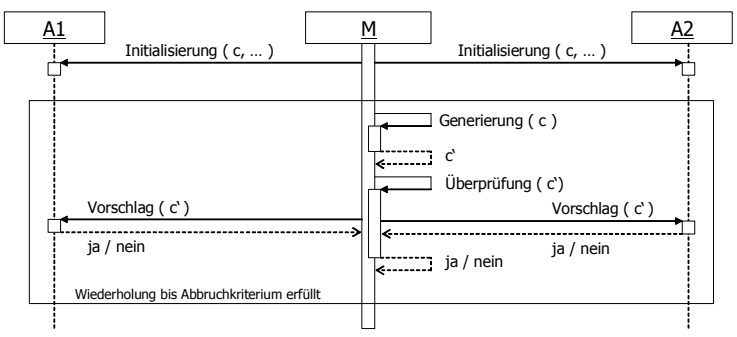

Abbildung 1: UML-Sequenzdiagramm des generischen Verhandlungsprozesses 


\subsection{Nähere Ausgestaltung des Verhandlungsprozesses}

In diesem Abschnitt werden aufbauend auf [Klei+02a] Ausprägungen der Variationspunkte des beschriebenen generischen Verhandlungsprozesses erörtert.

\subsubsection{Generierung von Vertragsvorschlägen}

Der Mediator kennt die Definition des Vertragsraums $C$, hat aber keine Informationen zu den Nutzenfunktionen der Verhandlungspartner. (Gängige Verfahren der multikriteriellen Optimierung sind damit nicht anwendbar.) Aufgrund der in der Regel vorliegenden Größe des Vertragsraums ist eine systematische Enumeration aller möglichen Vertragsalternativen nicht praktikabel. Demzufolge erscheint lediglich eine eingeschränkte Aufzählung einzelner Vertragsvorschläge möglich. Im einfachsten Fall bedeutet dies eine zufällige Generierung eines Vertrags aus $C$ auf Basis einer Gleichverteilung über den Vertragsraum. Unterstellt man, dass im Laufe des Verhandlungsprozesses der jeweils aktuell gültige Vertrag bereits eine relativ hohe Güte besitzt, so bietet sich eine zufallsbasierte Modifikation dieses Vertrags an. Eine solche Modifikation entspricht im Kontext lokaler Suchverfahren etwa einer zufälligen Auswahl einer so genannten Nachbarlösung (auf der Grundlage einer zu definierenden Nachbarschaftsrelation), während im Zusammenhang mit evolutionären (genetischen) Verfahren auch von einem Mutationsoperator gesprochen wird.

Auch wenn der Mediator keine Informationen zu der eigentlichen Güte der generierten und gegebenenfalls akzeptierten Vertragsvorschläge hat, sind indirekt eventuell gewisse Rückschlüsse möglich, so dass Formen des Lernens des Mediators einbezogen werden können. Beispielsweise kann eine Analyse des bisherigen Verhandlungsprozesses zu der Erkenntnis führen, dass Verträge mit einer bestimmten Ausprägung einer Attributkombination relativ häufig akzeptiert wurden; gegebenenfalls könnte der Mediator dann solche Attributausprägungen (zeitweise) fixieren. Vor dem Hintergrund evolutionärer Verfahren erscheint auch eine Rekombination von Fragmenten bereits akzeptierter Vertragsvorschläge als ein möglicher Generierungsmechanismus. Entsprechende Ansätze sind allerdings abhängig von der Definition des Vertragsraums.

\subsubsection{Akzeptanzkriterien der Verhandlungspartner}

Das „natürliche“ Akzeptanzkriterium eines Verhandlungspartners $A_{i}$ besteht darin, eine Veränderung eines bisher anerkannten Vertrags $c$ zu einem potenziellen Vertrag c' nur dann zu akzeptieren, wenn hiermit keine Verschlechterung des Nutzenwertes einhergeht (gieriges (greedy) Verhalten):

$$
A_{i}^{g}\left(c, c^{\prime}\right):= \begin{cases}\mathrm{Ja} & \text { falls } f_{i}\left(c^{\prime}\right) \geq f_{i}(c) \\ \text { Nein } & \text { sonst }\end{cases}
$$


Allerdings führt diese myopische Sichtweise selbst dann, wenn ein Akteur alleine den Vertrag diktieren könnte, letztendlich in der Regel nicht zu einem bestmöglichen Vertrag sondern allenfalls zu einem lokalen Optimum, da die zugrunde gelegten Vertragsraumstrukturen zumeist mit einem multimodalen Lösungsraum einhergehen (nur in Ausnahmefällen stellt ein ermitteltes lokales Optimum dann auch ein globales Optimum dar). Es ist zu vermuten, dass der Verhandlungsprozess um so mehr bei einer kombinierten Anwendung dieses Akzeptanzkriteriums relativ schnell ,ins Stocken“ käme, da eine beiderseitige Akzeptanz eines zufällig variierten Vertrags nach einigen Iterationen recht unwahrscheinlich wird.

Hinsichtlich dieser Problematik wurden für klassische Optimierungsproblemstellungen verschiedene Strategien (Metaheuristiken) entwickelt, die eine Überwindung lokaler Optima ermöglichen sollen. Im Zusammenhang mit einer zufallsbasierten Auswahl und Beurteilung von Nachbarlösungen werden im Rahmen von Simulated-Annealing-Strategien [Cern85; Kirk+83] mit einer gewissen Wahrscheinlichkeit auch Verschlechterungen akzeptiert. Zweckmäßigerweise erfolgt dies so, dass die Akzeptanzwahrscheinlichkeit im Laufe des Suchprozesses sowie mit steigendem Ausmaß der Verschlechterung abnimmt. Eine Übertragung des klassischen Akzeptanzkriteriums von Simulated Annealing (gemäß der so genannten Boltzmann-Verteilung bzw. -Funktion) führt unter Verwendung eines positiven Parameters $T$ (,Temperatur“) zu dem folgenden probabilistischen Akzeptanzkriterium eines Verhandlungspartners $A_{i}$ (kooperatives Verhalten):

$$
P\left(A_{i}^{k}\left(c, c^{\prime}\right)=\mathrm{Ja}\right):=\left\{\begin{array}{cl}
1 & \text { falls } f_{i}\left(c^{\prime}\right) \geq f_{i}(c) \\
e^{\left(f_{i}\left(c^{\prime}\right)-f_{i}(c)\right) / T} & \text { sonst }
\end{array}\right.
$$

Für $T \rightarrow 0$ gilt offensichtlich $A_{i}^{k} \rightarrow A_{i}^{g}$. Eine wesentliche Schwierigkeit besteht nun in der zweckmäßigen Wahl bzw. Reduktion des Parameters $T$ im Laufe des Verhandlungsprozesses. Eine mögliche Anforderung ist ein im Verhandlungsprozess jeweils möglichst gleich großer Kooperationsgrad - hinsichtlich der Häufigkeit des kurzfristig altruistischen Akzeptierens von Nutzenwertverschlechterungen - der beiden Verhandlungspartner. Dies stellt ein Problem dar, da prinzipiell beliebige Skalierungen der Nutzenfunktionen und Verteilungen der Nutzenwertveränderungen bei Vertragsmodifikation vorliegen können. Der hier entwickelte Lösungsansatz beruht auf der Vorgabe von Akzeptanzwahrscheinlichkeiten, wofür passende Parameterwerte und Anpassungsfunktionen bestimmt werden (unabhängig von einer positiv affinen Transformation der Nutzenfunktionen). Vorgegeben seien zwei Akzeptanzwahrscheinlichkeiten $p_{1}$ und $p_{2}, p_{1}>p_{2}$, für den Anteil der zu akzeptierenden verschlechternden Vertragsmodifikationen, der am Anfang bzw. am Ende des Verhandlungsprozesses näherungsweise erreicht werden soll. Die Ermittlung entsprechender Parameterwerte $T_{1}$ bzw. $T_{2}$ ist abhängig von dem Vertragsraum und der Nutzenfunktion und muss damit anwendungsspezifisch erfolgen. Hierzu simulieren die beiden Verhandlungspartner den Verhandlungsprozess für eine vorzugebende Anzahl an Verhandlungsrunden isoliert voneinander (,Pro- 
belauf"), wobei die Akzeptanzentscheidungen des jeweils nicht involvierten Verhandlungspartners zufallsbasiert auf der Grundlage der vorgegebenen Akzeptanzwahrscheinlichkeiten generiert werden. Man erhält hieraus eine Liste der aufgetretenen (negativen) Nutzenwertverschlechterungen $\left[\Delta_{1}, \Delta_{2}, \ldots, \Delta_{k}\right]$. Hiermit kann man den Erwartungswert der Anzahl der akzeptierten verschlechternden Vertragsmodifikationen mit dem vorgegebenen Anteil gleichsetzen:

$$
\sum_{i=1}^{k} e^{\Delta_{i} / T_{j}}=k \times p_{j} \quad \text { für } j=1,2
$$

Der Term auf der linken Seite der Gleichung sinkt mit wachsendem Parameterwert $T_{j}$ streng monoton. Die Gleichungen haben somit jeweils eine eindeutige Lösung. Die zu ermittelnden Parameterwerte $T_{j}$ können über ein iteratives Verfahren bestimmt werden (beispielsweise per Intervallhalbierung).

Eine gebräuchliche Form der Anpassung des Temperaturparameters $T$ im Verfahrensablauf von Simulated Annealing ist das so genannte geometrische Abkühlungsschema, was in Abhängigkeit von einem Abkühlungsparameter $\alpha, 0<\alpha<1$, nach jeder Verhandlungsrunde zur Anwendung kommt:

$$
T:=\alpha \times T
$$

Der Abkühlungsparameter $\alpha$ kann in Abhängigkeit von den ermittelten Temperaturparametern und von der Anzahl $r$ der Runden des Verhandlungsprozesses folgendermaßen bestimmt werden:

$$
\alpha:=\sqrt[r-11]{T_{2} / T_{1}}
$$

\subsection{Softwaretechnische Umsetzung}

Zur softwaretechnischen Umsetzung der vollautomatisierten Abwicklung des Verhandlungsprozesses können die Verhandlungspartner sowie der Mediator als softwarebasierte, autonome Agenten repräsentiert werden. Dabei könnten mobile Agenten der eigentlichen Verhandlungspartner auf eine gemeinsame Verhandlungsplattform mit dem Mediatoragenten migrieren und dort gemäß einem Interaktionsprotokoll autonom interagieren. Allerdings besitzt ein verteiltes System, bei dem die eigentliche Berechnung der Nutzenfunktion auf privaten Plattformen der Verhandlungspartner stattfindet, einen größeren Schutz vor einer Ausspähung der Nutzenfunktionen (über ein Reverse Engineering der Softwareagenten). Als universeller Kopplungsmechanismus bieten sich dann etwa Web Services an, wobei insbesondere ein XML-Nachrichtenformat zur Kodierung der Verträge zu definieren wäre. 
Zum Zwecke experimenteller Untersuchungen der beschriebenen Verhandlungsprozesse wurde in der Programmiersprache $\mathrm{C}++$ ein objektorientiertes Anwendungsframework entwickelt. Der hierin abgebildete generische Verhandlungsprozess kann für beliebige Vertragsräume $C$ sowie verschiedene Ausgestaltungen der in Abschnitt 2.1 beschriebenen Variationspunkte konkretisiert werden. Der Kern des Anwendungsframeworks ist in dem Klassendiagramm in Abbildung 2 in vereinfachter Form veranschaulicht. Die Akteure bzw. Agenten des Verhandlungsprozesses ergeben sich als Ausprägungen der generischen Klassen Mediator und Participant, die über den Vertragsraum $C$ sowie im ersten Fall außerdem durch den Mechanismus zur Generierung von Vertragsvorschlägen (Proposal) sowie die Regel zur letztlichen Anerkennung eines neues Vertragsvorschlags (Decision) statisch parametrisiert sind. Die zentrale Operation der Klasse Mediator setzt im Wesentlichen den in Abbildung 1 skizzierten Ablauf um. (Das Verhandlungs-Framework ist mit dem Metaheuristik-Framework HoTFrAME [Fink00] kompatibel, so dass Lösungsräume und Nachbarschaftsstrukturen aus HOTFRAME als Vertragsräume wiederverwendet werden können.)


Abbildung 2: UML-Klassendiagramm (vereinfacht) des Verhandlungs-Frameworks

\section{Experimentelle Untersuchung zur Ermittlung effektiver Koordinationsmechanismen}

Hinsichtlich einer zielgerichteten Gestaltung des Verhandlungsprozesses sind zunächst allgemeine Bewertungskriterien zu erörtern [Sand99]. Grundsätzlich ist anzustreben, dass der im Rahmen des Verhandlungsprozesses ermittelte Vertrag effizient (Pareto-optimal) ist. Die Forderung nach einer Maximierung des Gesamtnutzens beider Verhandlungspartner (soziale Wohlfahrt) setzt einen gemeinsamen Bewertungsmaßstab voraus (etwa über ein monetäres Äquivalent), was sich häufig als problematisch herausstellt. Im Weiteren wird ein Vertrag für einen Akteur insbesondere hinsichtlich der jeweiligen Abweichung von einem isoliert optimalen Vertrag bewertet (wobei dann zum Vergleich unterstellt wird, dass ein opportunis- 
tischer Akteur den Vertrag alleine diktieren könnte). Idealerweise ist ein Koordinationsmechanismus anreizkompatibel, was bedeutet, dass es für die Akteure rational ist, entsprechend ihrer wahren Nutzenfunktion zu handeln - ein Akteur kann sich dann also nicht durch Angabe falscher Informationen auf Kosten anderer besser stellen. Ein Koordinationsmechanismus sollte gewisse Fairnesskriterien dahingehend erfüllen, dass kein Akteur systematisch benachteiligt wird. Weitere Anforderungen sind die Geheimhaltung privater Informationen bzw. möglichst geringe Anforderungen an den Informationsstand sowie die berechungstechnische Praktikabilität des Koordinationsmechanismus.

Im Folgenden wird zunächst ein konkretes Anwendungsszenario entwickelt, anhand dessen verschiedene Ausgestaltungen des Verhandlungsprozesses experimentell miteinander verglichen werden.

\subsection{Beispielhaftes Anwendungsszenario}

Das im Weiteren beschriebene Anwendungsszenario kann als ein innerbetriebliches Steuerungsproblem aus der Produktionsplanung aufgefasst werden. Aufgrund der Komplexität der Steuerung von Produktionssystemen wird selbst im unternehmensinternen Umfeld unter gewissen Randbedingungen der Einsatz dezentraler bzw. marktlicher Koordinationsformen in Erwägung gezogen [Fink02]. Dies ist auch dadurch motiviert, dass in Unternehmen eine asymmetrische Informationsverteilung zu berücksichtigen ist und Akteure eigene Interessen verfolgen, die nicht ohne weiteres miteinander in Einklang gebracht werden können [MiRo92]. Ein Beispiel ist die Abstimmung zwischen einem Profit-Center Vertrieb, das sich an Erlösgesichtspunkten orientiert (etwa hinsichtlich der rechtzeitigen Fertigstellung von Kundenaufträgen) und einem Profit-Center Fertigung, das primär eine Kostenminimierung verfolgt (über eine entsprechende Zuordnung von Aufträgen zu Maschinen). Das hier im Rahmen dieses Szenarios betrachtete Maschinenbelegungsproblem ist hinsichtlich der resultierenden Verhandlungsproblemstellung auch für die zwischenbetriebliche Koordination zwischen per se unabhängigen Akteuren als beispielhaft anzusehen. Bezüglich der grundsätzlichen Aussagekraft ist weiterhin lediglich relevant, dass bereits die isolierte Ermittlung eines günstigen Vertrags für einen Akteur schwierig ist (mit Auswirkungen im Hinblick auf eine „Bounded Rationality“ im Sinne von [Simo82]) und damit umso mehr auch die verhandlungsbasierte Vertragsausgestaltung zwischen autonomen Akteuren mit konkurrierenden Zielen eine komplexe Aufgabe darstellt.

Es wird ein Maschinenbelegungsproblem mit Fließfertigungscharakteristik betrachtet (d.h. die Maschinenfolge der Arbeitsgänge aller Aufträge 1, ., $n$ ist identisch), wobei aus technischen Gründen eine kontinuierliche Fertigung eines jeden Auftrags auf den Maschinen 1, .., $m$ sicherzustellen ist; vgl. Abbildung 3. Entsprechende Problemstellungen treten insbesondere in der Prozessindustrie auf [Dude+92]. Die Bedingung der kontinuierlichen Fertigung führt dazu, dass die 
Fertigung (des ersten Arbeitsgangs) eines Auftrags $k$, der im Anschluss an einen Auftrag $i$ gefertigt wird, erst nach einer Mindestwartezeit $d_{i k}$ beginnen kann; diese Mindestwartezeiten können unter Betrachtung aller Arbeitsgangdauern $t_{i j}$ eines Auftrags $i$ auf Maschine $j$ berechnet werden. Aufgrund der Bedingung einer kontinuierlichen Fertigung ergibt sich auf allen Maschinen eine identische Auftragsfolge. Als Zielsetzung des Profit-Center Vertrieb sei die Minimierung der Summe der Fertigstellungszeitpunkte (Durchlaufzeiten) der Aufträge

$$
f_{1}(\Pi)=\sum_{i=2}^{n}(n+1-i) \cdot d_{\pi(i-1), \pi(i)}+\sum_{i=1}^{n} \sum_{j=1}^{m} t_{i j}
$$

über die Bestimmung einer Auftragspermutation $\Pi=(\pi(1), \ldots, \pi(n))$ zugrunde gelegt [FiVo03]. Diese Problemstellung ist $N P$-schwer [SaGo76].

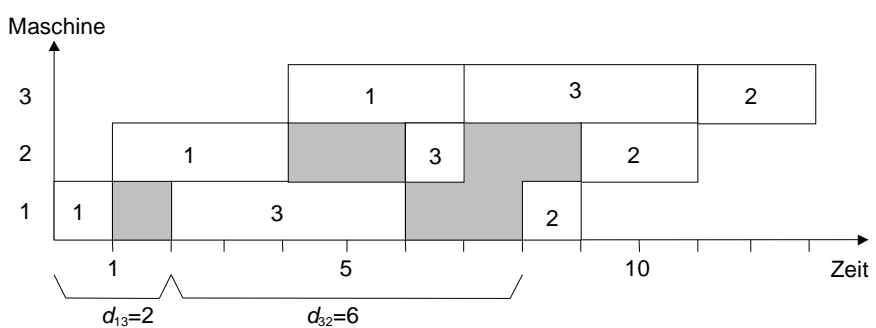

Abbildung 3: Beispiel für eine kontinuierliche Fließfertigung von Aufträgen

Unter der Berücksichtigung reihenfolgeabhängiger Rüstkosten $u_{i k}$, die sich ergeben, wenn Auftrag $k$ im Anschluss an Auftrag $i$ gefertigt wird (jeweils als Summe über alle Maschinen), resultiert eine hierzu konkurrierende Zielsetzung des ProfitCenter Fertigung als die Minimierung der beeinflussbaren Fertigungskosten

$$
f_{2}(\Pi)=\sum_{i=2}^{n} u_{\pi(i-1), \pi(i)}
$$

über die Bestimmung einer Auftragsauflage $\Pi=(\pi(1), \ldots, \pi(n))$. Diese Problemstellung ist als Variante des Traveling Salesman Problem ebenfalls $N P$-schwer.

Aus der Verhandlungsperspektive ist der Vertragsraum $C$ hier als die Menge aller möglichen Auftragsauflagen bzw. Permutationen $\Pi=(\pi(1), \ldots, \pi(n))$ der Aufträge $1, \ldots, n$ definiert. Dieser Vertragsraum umfasst damit beispielsweise bei $n=100$ Aufträgen $100 ! \approx 9 \times 10^{157}$ potenzielle Verträge. Die Aufgabe besteht nun darin, über eine Konkretisierung des Verhandlungsprozesses eine für beide Profit-Center möglichst gute gemeinsame Auftragsauflage als Vertrag zu bestimmen. Hierbei ist zu berücksichtigen, dass die beiden Zielsetzungen nicht direkt miteinander vergleichbar sind (Zeiten- vs. Kostenmaßstab) sowie ohnehin keine gemeinsame Information über die Zielfunktionen vorliegt. Außerdem ist bereits die isolierte Op- 
timierung einer Zielfunktion jeweils ein $N P$-schweres Problem und damit ab einer gewissen Problemgröße in der Regel nur noch heuristisch (approximativ) lösbar.

\subsection{Experimentelle Untersuchung}

Im Folgenden werden die beschriebenen Verhandlungsprozesse anhand des in Abschnitt 3.1 dargestellten Anwendungsszenarios experimentell untersucht, um hiermit normativ ausgerichtete Erkenntnisse zu einer möglichst effektiven Gestaltung entsprechender Verhandlungsprozesse zu gewinnen. Als Datengrundlage für das betrachtete Anwendungsszenario werden Scheduling-Datensätze aus der Literatur verwendet [Tai193]. Genutzt werden im Folgenden jeweils 30 Datensätze mit $n=100$ Aufträgen (ta061-ta090). Diese Datensätze wurden in [FiVo03] hinsichtlich der Zielsetzung der Minimierung der Summe der Durchlaufzeiten $\left(f_{1}(\Pi)\right)$ verwendet, so dass die dort heuristisch ermittelten besten Lösungen, die über die Berechnung unterer Schranken nachgewiesenermaßen im Mittel maximal 2\% von den Optimalwerten abweichen, als Referenzwerte verwendet werden können (hinsichtlich bestenfalls erreichbarer Nutzenwerte, wenn ein Akteur alleine den Vertrag diktieren könnte). Entsprechende Lösungen wurden auch für die Fertigungskostenzielsetzung $\left(f_{2}(\Pi)\right)$ ermittelt (unter Anwendung verschiedener Metaheuristiken - Iterated Steepest Descent, Simulated Annealing, Reactive Tabu Search - für jeweils bis zu 100 Sekunden). Die Rechenzeiten beziehen sich auf die Verwendung eines Pentium IV mit 1,8 GHz. Die Güte von Verträgen wird im Folgenden in der Regel über durchschnittliche prozentuale Abweichungen von den isoliert besten bekannten Lösungen (Verträgen) angegeben. Die Verhandlungspartner gehen jeweils von zwei voneinander unabhängigen Datensätzen aus. Ausgangspunkt der Verhandlungen ist die Identitätspermutation. Die zufallsbasierte Generierung neuer Vertragsvorschläge erfolgt über eine Verschiebung jeweils eines Auftrags an eine andere Position in der Auftragsfolge.

In den Tabellen 1 und 2 sind die Ergebnisse nach der jeweiligen Durchführung von 100.000 bzw. 1.000.000 Verhandlungsrunden bei einer Kombination verschiedener Akzeptanzkriterien der Verhandlungspartner dokumentiert. Bei kooperativen Agenten wurden zunächst die Parameterwerte $p_{1}=0,1$ und $p_{2}=0,01$ verwendet, wobei die Agenten jeweils Probeläufe mit 10\% der gesamten Verhandlungsrunden durchführen. Die Rechenzeit für einen Verhandlungsprozess mit 100.000 Runden beträgt etwa eine Sekunde sowie dementsprechend circa zehn Sekunden bei 1.000.000 Runden.

\begin{tabular}{|c|c|c|c|c|c|}
\hline \multirow{3}{*}{\multicolumn{2}{|c|}{$\begin{array}{l}\text { Resultate für: } \\
100.000 \text { Runden } \\
\text { p1=0,1; p2=0,01 }\end{array}$}} & \multicolumn{4}{|c|}{ Agent 2} \\
\hline & & \multicolumn{2}{|c|}{ Gierig } & \multicolumn{2}{|c|}{ Kooperativ } \\
\hline & & $f_{1}$ & $f_{2}$ & $f_{1}$ & $f_{2}$ \\
\hline \multirow{2}{*}{ Agent 1} & Gierig & $24,0 \%$ & $21,6 \%$ & $14,7 \%$ & $28,2 \%$ \\
\hline & Kooperativ & $29,6 \%$ & $14,8 \%$ & $17,8 \%$ & $19,2 \%$ \\
\hline
\end{tabular}

Tabelle 1: Jeweilige mittlere Abweichungen von den isoliert besten bekannten Verträgen nach Verhandlungsprozessen mit 100.000 Verhandlungsrunden 


\begin{tabular}{|c|c|c|c|c|c|}
\hline \multirow{3}{*}{\multicolumn{2}{|c|}{$\begin{array}{l}\text { Resultate für: } \\
\text { 1.000.000 Runden } \\
\text { p1=0,1;p2=0,01 }\end{array}$}} & \multicolumn{4}{|c|}{ Agent 2} \\
\hline & & \multicolumn{2}{|c|}{ Gierig } & \multicolumn{2}{|c|}{ Kooperativ } \\
\hline & & $f_{1}$ & $f_{2}$ & $f_{1}$ & $f_{2}$ \\
\hline \multirow{2}{*}{ Agent 1} & Gierig & $23,9 \%$ & $21,5 \%$ & $9,5 \%$ & $33,8 \%$ \\
\hline & Koopera & $32,7 \%$ & $10,5 \%$ & $13,3 \%$ & $16,8 \%$ \\
\hline
\end{tabular}

Tabelle 2: Jeweilige mittlere Abweichungen von den isoliert besten bekannten Verträgen nach Verhandlungsprozessen mit 1.000.000 Verhandlungsrunden

Die besten Gesamtergebnisse resultieren aus der Kombination zweier kooperativer Verhandlungspartner. Bei 1.000.000 Verhandlungsrunden ergibt sich etwa eine mittlere Abweichung von circa $15 \%$. Nun stellt sich zunächst insbesondere die Frage, wie weit diese mittlere Abweichung theoretisch reduziert werden könnte, wenn herkömmliche Optimierungsverfahren direkt auf einer Gesamtzielfunktion $f(\Pi)$ operieren könnten. Dabei sei $f(\Pi)$ definiert als die normiert gewichtete Summe von $f_{1}(\Pi)$ und $f_{2}(\Pi)$ dergestalt, dass letztlich die mittlere prozentuale Abweichung von den besten bekannten isolierten Verträgen bewertet wird. Für diese Gesamtzielfunktion wurden verschiedene Metaheuristiken - Iterated Steepest Descent, Simulated Annealing, Reactive Tabu Search - mit einer Rechenzeit von jeweils zehn Sekunden (entsprechend der Rechenzeit beim Verhandlungsprozess mit 1.000.000 Verhandlungsrunden) angewendet. Hierbei ergab sich eine mittlere Abweichung des Verhandlungsergebnisses vom jeweils besten Ergebnis der integrierten Optimierung von nur circa 2\%. In Anbetracht der stark eingeschränkten Informationsverfügbarkeit im Rahmen der Steuerung des Verhandlungsprozesses - da die Verhandlungspartner ihre jeweiligen Nutzenfunktionen nicht offen legen - ist dieses Ergebnis in positiver Hinsicht als überraschend zu bewerten.

Der Vergleich der Ergebnisse der Kombination zweier gieriger Verhandlungspartner bei 100.000 Verhandlungsrunden und 1.000.000 Verhandlungsrunden bestätigt die Vermutung, dass der Verhandlungsprozess relativ schnell ,ins Stocken“ kommt. Damit kann eine Verlängerung des Verhandlungsprozesses keinen wesentlichen Erfolg bringen, da eine beiderseitige Akzeptanz eines zufällig variierten Vertrags relativ schnell sehr unwahrscheinlich wird (lokales Vertragsoptimum). Die Abweichungen bei der Kombination zweier kooperativer Verhandlungspartner dominieren die Abweichungen bei der Kombination zweier gieriger Verhandlungspartner elementweise, so dass die Kombination zweier gieriger Verhandlungspartner eine dominierte Variante darstellt (vgl. aber Abschnitt 4).

Eine Betrachtung von Kombinationen zweier Verhandlungspartner mit unterschiedlichen Akzeptanzkriterien zeigt, dass in solchen Fällen ein gieriger Verhandlungspartner auf Kosten des kooperativen Verhandlungspartners sehr gute Verträge erzielen kann. Dementsprechend sind die Ergebnisse der Kombination zweier Verhandlungspartner mit unterschiedlichen Akzeptanzkriterien effizient (Pareto-optimal). Das Ungleichgewicht verstärkt sich bei einer Verlängerung des Verhandlungsprozesses etwa von circa $29 \%$ vs. $15 \%$ zu $33 \%$ vs. $10 \%$. Dies ist plausibel, da im letzteren Fall der kooperative Verhandlungspartner eine längere 
Zeitspanne mit hohen Akzeptanzwahrscheinlichkeiten operiert, was von dem gierigen Verhandlungspartner in seinem Sinne ausgenutzt werden kann.

Die Erklärung der Ergebnisse steht im Zusammenhang mit den in den Tabellen 3 und 4 dokumentierten Akzeptanzhäufigkeiten in Abhängigkeit von den Akzeptanzkriterien der Verhandlungspartner. So ist eine Anerkennung eines Vertragsvorschlags - das heißt ein simultanes Akzeptieren durch beide Verhandlungspartner - bei 1.000.000 Verhandlungsrunden bei zwei gierigen Verhandlungspartnern extrem unwahrscheinlich, obwohl beide Verhandlungspartner isoliert relativ viele Vertragsvorschläge mit Nutzenzuwachs bewerten.

\begin{tabular}{l|l|c|c|cccc} 
Agent 1 & Agent 2 & 1:Ja & 2:Ja & 1:Ja / 2:Ja & 1:Ja / 2:Nein & 1:Nein / 2:Ja & 1:Nein / 2:Nein \\
\hline Gierig & Gierig & $7,43 \%$ & $5,75 \%$ & $0,13 \%$ & $7,30 \%$ & $5,63 \%$ & $86,95 \%$ \\
Gierig & Kooperativ & $2,88 \%$ & $13,70 \%$ & $0,19 \%$ & $2,69 \%$ & $13,51 \%$ & $83,61 \%$ \\
Kooperativ & Gierig & $14,81 \%$ & $2,57 \%$ & $0,17 \%$ & $14,64 \%$ & $2,40 \%$ & $82,79 \%$ \\
Kooperativ & Kooperativ & $7,50 \%$ & $7,15 \%$ & $0,38 \%$ & $7,12 \%$ & $6,77 \%$ & $85,73 \%$
\end{tabular}

Tabelle 3: Akzeptanzhäufigkeiten und entsprechende Kombinationen im Verhandlungsprozess bei 100.000 Verhandlungsrunden

\begin{tabular}{l|l|c|c|cccc} 
Agent 1 & Agent 2 & 1:Ja & 2:Ja & 1:Ja / 2:Ja & 1:Ja / 2:Nein & 1:Nein / 2:Ja & 1:Nein / 2:Nein \\
\hline Gierig & Gierig & $6,59 \%$ & $5,03 \%$ & $0,01 \%$ & $6,57 \%$ & $5,01 \%$ & $88,40 \%$ \\
Gierig & Kooperativ & $0,72 \%$ & $19,68 \%$ & $0,02 \%$ & $0,70 \%$ & $19,66 \%$ & $79,62 \%$ \\
Kooperativ & Gierig & $19,12 \%$ & $0,82 \%$ & $0,02 \%$ & $19,09 \%$ & $0,80 \%$ & $80,09 \%$ \\
Kooperativ & Kooperativ & $5,97 \%$ & $6,56 \%$ & $0,22 \%$ & $5,75 \%$ & $6,34 \%$ & $87,68 \%$
\end{tabular}

Tabelle 4: Akzeptanzhäufigkeiten und entsprechende Kombinationen im Verhandlungsprozess bei 1.000.000 Verhandlungsrunden

Die iterative Ausgestaltung von Verträgen ist in Abbildung 4 hinsichtlich der sukzessiven Nutzenverbesserung veranschaulicht. Dargestellt sind die mittleren Abweichungen der beiden Verhandlungspartner im Laufe des Verhandlungsprozesses. Ein gieriger Verhandlungspartner kann in Kombination mit einem kooperativen Verhandlungspartner offensichtlich insbesondere im ersten Bereich des Verhandlungsprozesses den Vertrag weitgehend in seinem Sinne beeinflussen, da der kooperative Verhandlungspartner dann relativ bereitwillig auch eigene Verschlechterungen in Kauf nimmt. Hier wie auch bei dem eher gleichförmigen Verlauf bei zwei kooperativen Verhandlungspartnern ergeben sich im späteren Bereich des Verhandlungsprozesses praktisch keine messbaren Verbesserungen mehr. Demzufolge bietet sich die Untersuchung der Verwendung erhöhter Akzeptanzwahrscheinlichkeiten an. 

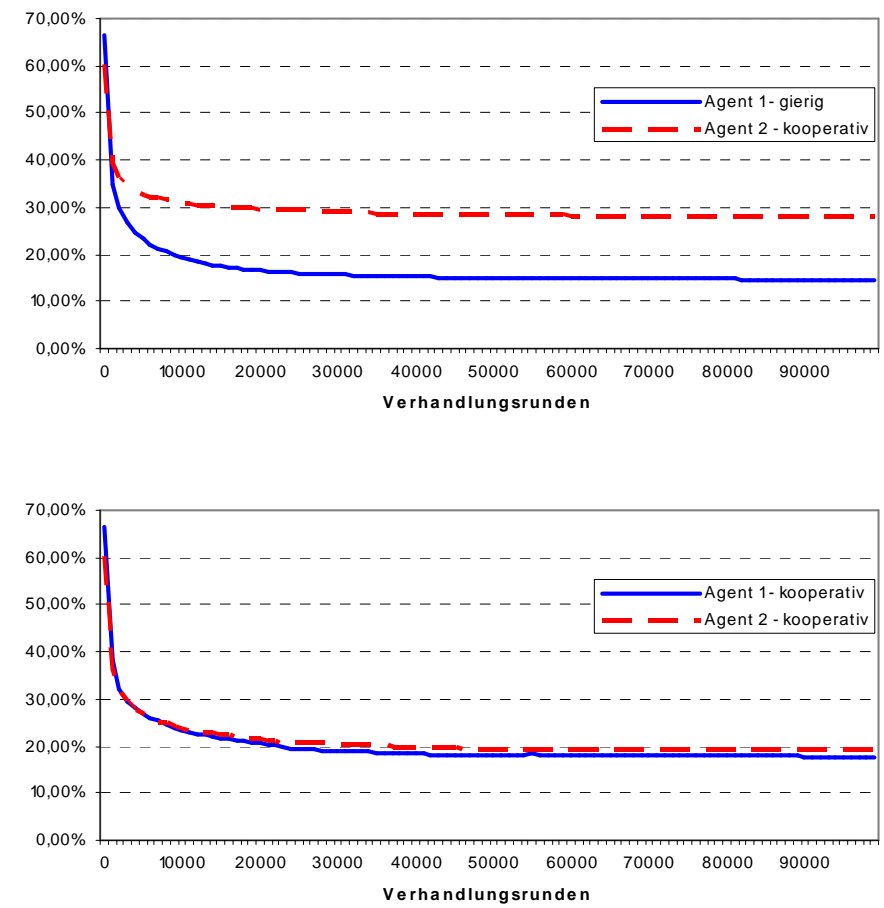

Abbildung 4: Entwicklung der mittleren Abweichungen von den isoliert besten bekannten Verträgen im Laufe von Verhandlungsprozessen zwischen einem gierigen und einem kooperativen Verhandlungspartner bzw. zwei kooperativen Verhandlungspartnern

Bei den bisher dargestellten Ergebnissen wurde bei kooperativen Verhandlungspartnern von angestrebten Akzeptanzwahrscheinlichkeiten $p_{1}=0,1$ (am Anfang des Verhandlungsprozesses) und $p_{2}=0,01$ (am Ende des Verhandlungsprozesses) ausgegangen. Über eine Experimentreihe wurde die Abhängigkeit der letztlich erzielten Vertragsgüte - bei Verhandlungsprozessen mit zwei kooperativen Verhandlungspartnern - von Akzeptanzwahrscheinlichkeiten untersucht. Der Mittelwert der durchschnittlichen prozentualen Abweichungen der beiden Verhandlungspartner ist in Abbildung 5 in Abhängigkeit von verschiedenen Akzeptanzwahrscheinlichkeitskombinationen dargestellt. Die besten Ergebnisse wurden jeweils für die Kombination $p_{1}=0,2$ und $p_{2}=0,04$ erzielt, wobei letztendlich nur noch eine etwa einprozentige Abweichung von dem besten Ergebnis der integrierten Optimierung einer Gesamtzielfunktion auftritt. 

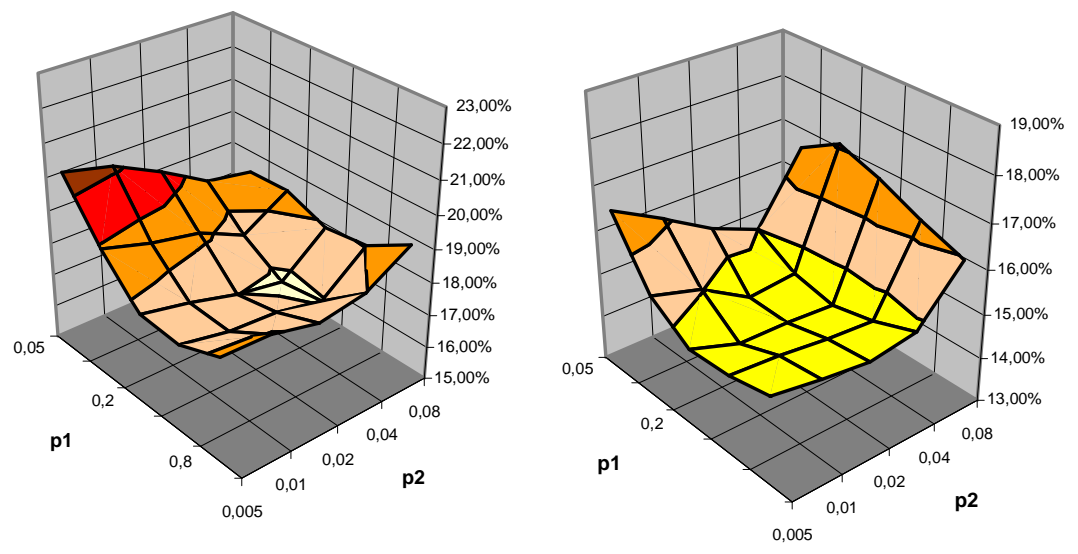

Abbildung 5: Durchschnittliche Vertragsgüte bei Verhandlungsprozessen mit zwei kooperativen Verhandlungspartnern in Abhängigkeit von den Parameterwerten $p_{1}$ und $p_{2}$ bei 100.000 Verhandlungsrunden (links) bzw. 1.000.000 Verhandlungsrunden (rechts)

\section{Spieltheoretische Analyse}

Im vorausgehenden Abschnitt wurden Verhandlungsergebnisse bei der Kombination von Verhandlungspartnern mit verschiedenen Strategien (Akzeptanzkriterien) untersucht und miteinander verglichen, ohne hierbei eine Aussage zu der eigentlichen Strategiewahl eines Akteurs zu machen. Hierzu ist eine spieltheoretische Betrachtung des Zusammenwirkens von Entscheidungskalkülen mehrerer rational handelnder Akteure erforderlich. Da den Akteuren jeweils bewusst ist, dass ihre Entscheidungen (bzw. die entsprechenden Handlungen) wechselseitig voneinander abhängig sind, sind in den Entscheidungskalkülen entsprechende Interdependenzen zu berücksichtigen, was jeweils mit Erwartungen zu den Verhaltensweisen der anderen Akteure verbunden ist. Man spricht dann auch von einer strategischen Entscheidungssituation bzw. einem strategischen Verhalten von Akteuren. Bei den so genannten nicht-kooperativen Spielen wird ein Akteur im Rahmen vorgegebener und durchsetzbarer Regeln auf Basis rationaler Erwägungen opportunistisch seinen Eigennutzen zu maximieren versuchen. Hierbei sind Zusicherungen zum eigenen Verhalten nur dann bindend, wenn bestimmte Institutionen eine Einhaltung verifizieren und durchsetzen können.

Unter diesen Annahmen ist die in den Tabellen 1 bzw. 2 dargestellte Entscheidungssituation zu betrachten, wobei prinzipiell für jeden Akteur die Wahl zwischen einem gierigen oder kooperativen Handeln besteht. Ein Akteur kann nun für jede Handlungsoption des anderen Akteurs seine beste Strategie bestimmen. So- 
fern der zweite Akteur gierig handelt, wird der erste Akteur über ein gieriges Verhandeln das für sich beste Resultat erzielen. Wird sich der zweite Akteur kooperativ verhalten, so führt für den ersten Akteur ebenfalls ein gieriges Verhandeln zum besten Resultat. Das heißt, unabhängig vom Verhalten des Anderen stellt für einen Akteur die gierige Verhandlungsstrategie die eindeutig beste „Antwort“ dar. Auf diese Weise ergibt sich eine Ausprägung des klassischen Gefangenendilemmas, da sich beide Akteure gierig verhalten werden und damit letztlich eine Lösung (NashGleichgewicht) resultiert, die hinsichtlich der jeweils erzielten Nutzenwerte von der Lösung, bei der sich beide Akteure kooperativ verhalten, dominiert wird [Klei+02a]. Dies erscheint auf den ersten Blick paradox, da sich hiermit beide Akteure schlechter stellen als bei einem beiderseitigen kooperativen Verhalten - aufgrund der gegebenen Spielregeln ist das Resultat aber unausweichlich, wenn man die Prämissen nicht-kooperativer Spiele ernst nimmt (rationale Eigennutzenüberlegungen ohne die Möglichkeit für bindende Abmachungen). Im Weiteren werden verschiedene Auswege aus dieser unbefriedigenden Situation diskutiert.

Zum einen kann man einen Verhandlungsprozess als ein so genanntes mehrstufiges Spiel betrachten, in dem die Akteure vor jeder Verhandlungsrunde adaptiv eine neue Entscheidung über das anzuwendende Akzeptanzkriterium treffen. In solchen Spielen kann unter gewissen Bedingungen ein dauerhaft kooperatives Verhalten in der Tat auch rational-eigennützig die beste Strategie darstellen [Axel84]. Voraussetzung hierfür ist das Erkennen eines nicht-kooperativen Verhaltens des anderen Akteurs, was in dem hier betrachteten Anwendungsfall erst nach etlichen Verhandlungsrunden feststellbar sein wird. Allerdings wird gerade in diesen ersten Verhandlungsrunden ein gieriger Verhandlungspartner bereits einen Großteil des möglichen Kooperationserfolges für sich sichern können (vgl. Abbildung 4), weshalb das Dilemma prinzipiell bestehen bleibt [Klei+02a].

Ein anderer Ansatz zur Auflösung von Gefangenendilemmasituationen ist die Veränderung der „Spielregeln“ hinsichtlich der Sicherung potenzieller Kooperationsvorteile. Eine solche Möglichkeit besteht darin, das probabilistische Akzeptanzkriterium statt bei den Agenten direkt beim Mediator anzusiedeln [Klei+02b]. Dies setzt einerseits Antworten der Verhandlungspartner voraus, die nicht nur einfache Ja- bzw. Nein-Angaben darstellen, sondern auch das Ausmaß der Verschlechterung angeben oder zu einem gewissen Grad andeuten. Damit werden mehr Informationen offen gelegt, die in einem komplizierteren und damit gegebenenfalls weniger robusten Ablauf verarbeitet werden. Trotzdem ist die resultierende Vertragsgüte in den Untersuchungen von [Klei+02b] schlechter als zuvor.

Ein einfacher und effektiver Ansatz zur Lösung des Dilemmas ist die Vorgabe (und Verifikation) des Kooperationsgrades in der Form von Mindestakzeptanzhäufigkeiten im Laufe des Verhandlungsprozesses. Die in Abschnitt 3 eingeführten Akzeptanzwahrscheinlichkeiten $p_{1}$ und $p_{2}$ eignen sich im Zusammenhang mit dem gewählten geometrischen Abkühlungsschema direkt zur Ableitung kumulierter Mindestakzeptanzhäufigkeiten über den Verhandlungsverlauf. Ein Verhandlungsprozess wird vom Mediator abgebrochen, sobald ein Akteur entsprechende Gren- 
zen unterschreitet (was jeweils nach einer gewissen Rundenanzahl erneut überprüft wird), so dass die Akteure nur bei Einhaltung der Vorgaben Kooperationsvorteile über entsprechend zustande gekommene Verträge erzielen können. Dieser Ansatz geht einher mit einem hohen Maß an Fairness, da beide Verhandlungspartner gleich viele Vertragsvorschläge akzeptieren müssen. Die typische Entwicklung von Akzeptanzhäufigkeiten für die jeweils bisher absolvierten Verhandlungsrunden ist in Abbildung 6 veranschaulicht.
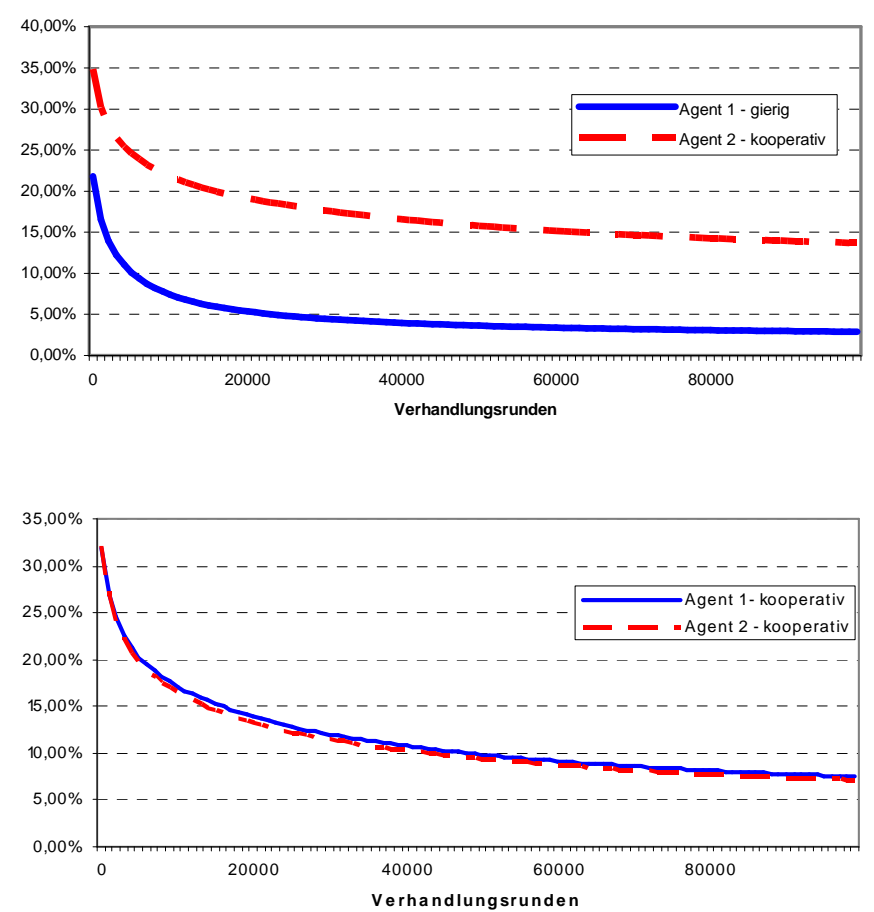

Abbildung 6: Entwicklung von Akzeptanzhäufigkeiten für die jeweils bisher absolvierten Verhandlungsrunden im Laufe von Verhandlungsprozessen zwischen einem gierigen und einem kooperativen Verhandlungspartner bzw. zwei kooperativen Verhandlungspartnern

Trotz dieser relativ strikten Vorgaben leidet die Güte der resultierenden Verträge nicht, da exakt die in Abschnitt 3 beschriebenen Mechanismen zur Anwendung kommen können. Es ist jedoch zu hinterfragen, ob die wahrheitsgemäße Anwendung des kooperativen Akzeptanzkriteriums für die Akteure in der Tat ein anreizkompatibles Verhalten darstellt. Eine systematisch nicht-wahrheitsgemäße Anwendung des vordefinierten Akzeptanzkriteriums würde bedeuten, dass ein Akteur teilweise schlechtere Vertragsvorschläge auf Kosten besserer Vertragsvorschläge mit einer höheren Wahrscheinlichkeit akzeptieren müsste, so dass die Anreizkom- 
patibilität plausibel erscheint. Ein formaler Beweis stellt sich allerdings als schwierig dar, da im Allgemeinen quasi gezeigt werden müsste, dass die Boltzmann-Funktion zu einem bestmöglichen Akzeptanzkriterium führt.

\section{Zusammenfassung und Ausblick}

In dem Beitrag wurden aufbauend auf [Klei+02a] automatisierte Koordinationsmechanismen zur Ausgestaltung komplexer Verträge im Rahmen mediatisierter Verhandlungsprozesse betrachtet. Neuartig ist insbesondere der beschriebene allgemeine Mechanismus zur fairen Konkretisierung der Akzeptanzkriterien kooperativer Verhandlungspartner für beliebige Vertragsräume und Nutzenfunktionen. Verhandlungsprozesse wurden anhand einer komplexen realitätsbezogenen Problemstellung experimentell untersucht. Trotz der Allgemeingültigkeit des Verhandlungsprotokolls, grundsätzlicher Informationsmängel sowie strategisch agierender Verhandlungspartner konnten hierbei in effizienter Form Verhandlungsergebnisse erzielt werden, die hinsichtlich der sozialen Wohlfahrt lediglich circa $1 \%$ von den Ergebnissen einer hypothetischen Optimierung einer expliziten Gesamtzielfunktion abweichen. Die beschriebenen Verhandlungsprozesse können damit unter der Voraussetzung einer formalen Definition des Vertragsraums einen effektiven Mechanismus zur Koordination autonomer Akteure mit privaten Informationen darstellen. Anwendungspotenziale bestehen sowohl bei der unternehmensinternen als auch bei der unternehmensübergreifenden Koordination.

Für die zukünftige Forschung ergeben sich eine Reihe interessanter Fragestellungen. Zunächst sind die Variationspunkte des Verhandlungsprozesses differenzierter zu betrachten. Dies bezieht sich etwa auf andersartige Akzeptanzkriterien bei den Verhandlungspartnern und beim Mediator. Weiterhin ist das angedeutete Lernpotenzial des Mediators für eine zielgerichtete Vertragsgenerierung zu konkretisieren und zu untersuchen; außerdem könnte auch ein Beitrag der Verhandlungspartner zur Generierung von Vertragsvorschlägen in Betracht gezogen werden. Generell ist zu untersuchen, unter welchen Bedingungen die Kombination kooperativer Verhandlungspartner die beste Konfiguration darstellt. Geht man davon aus, dass dies nicht immer gilt, so ergibt sich bei spontanen Vertragsverhandlungen das Problem der strategischen Wahl von Akzeptanzkriterien durch die Verhandlungspartner unter unvollständigen Informationen. Die Möglichkeit der Erweiterung des beschriebenen Verhandlungsprozesses von bilateralen auf multilaterale Verhandlungen ist offensichtlich. Ein Beispiel ist die Verhandlung von Lieferverträgen zwischen einem Lieferanten, einem Logistikdienstleister und einem Abnehmer. Im Allgemeinen stellt sich die Aufgabe der zweckmäßigen Standardisierung und Formalisierung von Vertragsräumen in typischen Geschäftsbeziehungen. Letztlich ist zu betonen, dass die beschriebenen Verhandlungsprozesse im Rahmen eines übergeordneten Matching zwischen Akteuren auf einem Markt- 
platz zum Einsatz kommen können. Geht man etwa von Akteuren in zwei zueinander komplementären Rollen aus, so kann zunächst für alle komplementären Kombinationen ein potenzieller Kooperationserfolg über eine jeweilige Verhandlung bestimmt werden. (Die Menge der zu führenden Verhandlungen ist quadratisch in der Anzahl der Akteure und macht damit eine Vollautomation praktisch unabdingbar.) Die sich ergebende Affinitätsmatrix führt gegebenenfalls zu einem effizient lösbaren linearen Zuordnungsproblem.

\section{Literatur}

[Axel84] Axelrod, R.: The Evolution of Cooperation. Basic Books: New York, 1984.

[BaBr93] Bakos, J. Y.; Brynjolfsson, E.: Information technology, incentives, and the optimal number of suppliers. Journal of Management Information Systems 10 (2), 1993: S. $37-54$.

[BeSe97] Beam, C.; Segev, A.: Automated negotiations: A survey of the state of the art. Wirtschaftsinformatik 39, 1997: S. 263-268.

[Bich+02] Bichler, M.; Kersten, G. E.; Strecker, S.: Towards a structured design of electronic negotiations. InterNeg Reports INR07/02, 2002.

[Cern85] Cerný, V.: Thermodynamical approach to the traveling salesman problem: An efficient simulation algorithm. Journal of Optimization Theory and Applications 45, 1985: S. 41-51.

[Clem+93] Clemons, E. K.; Reddi, S. P.; Row, M. C.: The impact of information technology on the organization of economic activity: the "move to the middle" hypothesis. Journal of Management Information Systems 10 (2), 1993: S. 9-36.

[Dude+92] Dudek, R. A.; Panwalkar, S. S.; Smith, M. L.: The lessons of flowshop scheduling research. Operations Research 40, 1992: S. 7-13.

[Fati+03] Fatima, S. S.; Wooldridge, M.; Jennings, N. R.: Optimal agendas for multi-issue negotiation. In: Proceedings of the Second International Conference on Autonomous Agents and Multiagent Systems (AAMAS-03), Melbourne, Australien, 2003.

[Fink00] Fink, A.: Software-Wiederverwendung bei der Lösung von Planungsproblemen mittels Meta-Heuristiken. Shaker: Aachen, 2000.

[Fink02] Fink, A.: Grenzen des Einsatzes einer agentenbasierten Produktionsplanung mit marktorientierten Koordinationsmechanismen. PPS Management 7, 2002: S. 47-50.

[FiVo03] Fink, A.; Voß, S.: Solving the continuous flow-shop scheduling problem by metaheuristics. European Journal of Operational Research, im Druck, 2003.

[Jenn+01] Jennings, N. R.; Faratin, P.; Lomuscio, A. R.; Parsons, S.; Woolridge, M.; Sierra, C.: Automated negotiation: Prospects, methods and challenges. Group Decision and Negotiation 10, 2001: S. 199-215.

[Kers+00] Kersten, G. E.; Noronha, S. J.; Teich, J.: Are all e-commerce negotiations auctions? Fourth International Conference on the Design of Cooperative Systems (COOP'2000), Sophia-Antipolis, France, May 23-26, 2000. 
[Kers02] Kersten, G. E.: The science and engineering of E-negotiation: Review of the emerging field, InterNeg Reports INR05/02, Draft paper, October 5, 2002.

[Kers03] Kersten, G. E.: The science and engineering of E-Negotiation: An introduction. Proceedings of the 36th Hawaii International Conference on Systems Sciences. IEEE Computer Society Press: Los Alamitos, 2003 (CD ROM, 10 Seiten).

[Kirk+83] Kirkpatrick, S., Gelatt Jr., C. D.; Vecchi, M. P.: Optimization by simulated annealing. Science 220, 1983: S. 671-680.

[Klei+02a] Klein, M.; Faratin, P.; Sayama, H.; Bar-Yam, Y.: Negotiating complex contracts. Proceedings of the First International Joint Conference on Autonomous Agents and Multiagent Systems (AAMAS'02), ACM Press: New York, 2002, S. 753-757.

[Klei+02b] Klein, M.; Faratin, P.; Bar-Yam, Y.: Using an annealing mediator to solve the prisoners' dilemma in the negotiation of complex contracts. In: Fourth Workshop on Agent-mediated Electronic Commerce (AMEC-IV), Lecture Notes in Artificial Intelligence 2531, Springer: Berlin Heidelberg, 2002, S. 194-202.

[Krau01] Kraus, S.: Strategic Negotiation in Multiagent Environments. MIT Press: Cambridge MA, 2001.

[Lomu+03] Lomuscio, A. R.; Wooldridge, M.; Jennings, N. R.: A classification scheme for negotiation in electronic commerce. Group Decision and Negotiation 12, 2003, S. 3156.

[Mert95] Mertens, P.: Wirtschaftsinformatik: Von den Moden zum Trend. In: König, W. (Hrsg.) Wirtschaftsinformatik '95. Physica: Heidelberg, 1995, S. 25-64.

[MiRo92] Milgrom, P.; Roberts, J.: Economics, Organization \& Management. Prentice Hall: Englewood Cliffs, 1992.

[Muth99] Muthoo, A.: Bargaining Theory with Applications. Cambridge University Press: Cambridge MA, 1999.

[Nape02] Napel, S.: Bilateral Bargaining - Theory and Applications. Lecture Notes in Economics and Mathematical Systems 518. Springer: Berlin Heidelberg, 2002.

[Nash50] Nash, J. F.: The bargaining problem. Econometrica 18, 1950: S. 155-162.

[Nash53] Nash, J. F.: Two-person cooperative bargaining games. Econometrica 21, 1953 : S. $128-140$.

[Pete00] Peters, R.: Elektronische Märkte und automatisierte Verhandlungen. Wirtschaftsinformatik 42, 2000: S. 413-421.

[Raif82] Raiffa, H.: The Art and Science of Negotiation. Harvard University Press: Cambridge MA, 1982.

[Rebs01] Rebstock, M.: Elektronische Unterstützung und Automatisierung von Verhandlungen. Wirtschaftsinformatik 43, 2001: S. 609-617.

[Roth85] Roth, A. E. (Hrsg.): Game-theoretic Models of Bargaining. Cambridge University Press: Cambridge MA, 1985.

[RoZ194] Rosenschein, J. S.; Zlotkin, G.: Rules of Encounter - Designing Conventions for Automated Negotiation among Computers. MIT Press: Cambridge MA, 1994.

[Rubi82] Rubinstein, A.: Perfect equilibrium in a bargaining model. Econometrica 50, 1982: S. 97-109. 
[SaGo76] Sahni, S.; Gonzales, T.: P-complete approximation problems. Journal of the Association of Computing Machinery 23, 1976: S. 555-565.

[Sand99] Sandholm, T. W.: Distributed and rational decision making. In: Weiss, G. (Hrsg.) Multiagent Systems: A Modern Approach to Distributed Artificial Intelligence. MIT Press: Cambridge MA, 1999, S. 201-258.

[Simo82] Simon, H. A.: Models of Bounded Rationality. MIT Press: Cambridge MA, 1982.

[Strö02] Ströbel, M.: Engineering Electronic Negotiations. Kluwer Academic Publishers: New York, 2002.

[Tai193] Taillard, E. D.: Benchmarks for basic scheduling instances. European Journal of Operational Research 64, 1993: S. 278-285.

[VoSc02] Voß, S.; Schneidereit, G.: Interdependencies between supply contracts and transaction costs. In: Seuring, S.; Goldbach, M. (Hrsg.) Cost Management in Supply Chains. Springer: Berlin Heidelberg, 2002, S. 255-274.

[Wede00] Wedekind, H.: On specifying contract negotiations. In: Hansen, H.-R.; Bichler, M.; Mahrer, H. (Hrsg.) Proceedings of the $8^{\text {th }}$ European Conference on Information Systems (ECIS 2000), Volume 1, Wien, 2000, S. 23-30. 